\title{
Wer versorgt uns morgen?
}

\begin{abstract}
Hartmannbund widmet sich Zukunftsfragen. Über Perspektiven neuer interdisziplinärer und intersektoraler Versorgungsmodelle sowie Chancen und Auswirkungen der Digitalisierung im Gesundheitswesen haben Experten bei der Jahreshauptversammlung des Hartmannbundes diskutiert. Intensiv setzten sie sich auch mit der Bedeutung neuer Gesundheitsberufe und deren künftiger Rolle in der Versorgung auseinander.
\end{abstract}

AUTORIN: SABINE SCHMITT

Mensch oder Roboter? Wohin geht die Reise in der medizinischen Versorgung? Dass es höchste Zeit wird, Veränderungen ins Auge zu sehen, machte Professor Dr. Boris Augurzky in seinem Impulsvortrag deutlich, den er "Zwischen Dampfmaschine und Raumschiff Enterprise" genannt hat. Angefangen von der Mission „Alpha Centrauri“ über künstliche Intelligenz und Robotik bis hin zu Genom-Operationen sei heute bereits vieles möglich - und könnte auch in der medizinischen Versorgung eingesetzt werden. Allerdings: „Das deutsche Gesundheitswesen macht es sich in der Steinzeit noch recht bequem", konstatierte der Volkswirt und Mathematiker
Augurzky, der am RWI - Leibnizinstitut für Wirtschaftsforschung lehrt. Innovationen gebe es viele, doch „dummerweise kommen diese beim Patienten nicht an“. Der Veränderungsdruck allerdings wächst laut Augurzky stark. Doch noch seien durch die hohe Regulierungsdichte des Gesundheitssystems Blockaden aus den Reihen der sogenannten Innovationsverlierer an der Tagesordnung. „Deshalb kommt das Gesundheitssystem gerade im Bereich Digitalisierung, wie bei elektronischer Patientenakte, Versorgungsvernetzung und vereinfachter Nutzung von Daten, nur im Schneckentempo voran“, erläuterte der Wissenschaftler. Spätestens im

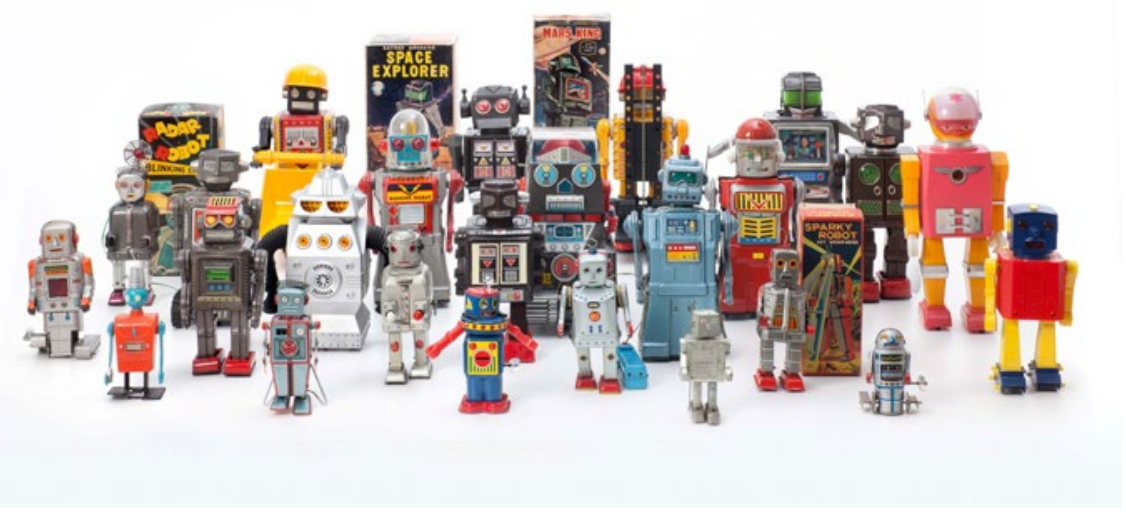

nächsten Jahrzeht werde sich das System anpassen müssen, um die Versorgung aufrechterhalten zu können.

\section{„ÄRZTE WERDEN MEHR DELEGIEREN MÜSSEN"}

$\mathrm{Ob}$ zu diesen Veränderungen tatsächlich Drohnen gehören werden, die beispielsweise Blutproben ins Labor fliegen, ob Versorgungsassistenten, die mit Laptops zu den Patienten reisen und den Arzt bei Bedarf online zuschalten oder ob sprachgestützte Homeassistenzsysteme künftig zum wichtigen Gesundheitsberater werden, kann heute noch nicht vorhergesagt werden, denn Prognosen haben die Neigung, völlig in die Irre zu gehen. „Was allerdings klar ist: Es werden andere Berufsbilder in der Zukunft benötigt, Ärzte werden mehr delegieren müssen, und die Differenzierung innerhalb der medizinischen Berufe wird größer werden“, betont Augurzky. Seine Mitdiskutanten auf dem Podium berichteten unter anderem von Best-Practice-Beispielen, in denen die Zukunft bereits in der Versorgung angekommen ist.

\section{ROBOTER ALS NACHT- UND STATIONSWACHE}

Seine Schöpfung zur Betreuung von demenzkranken Patienten hatte Professor Dr. Hans-Joachim Böhme von der Universität Dresden gleich mitgebracht: „Anna-Constantia“ ist ein künstlich intelligenter Roboter, der in einem Modellversuch als Nacht- oder Stationswache in einer Einrichtung für Demenzpatienten mitarbeitet. „Wir erforschen, was mit solchen Systemen im klinischen und therapeutischen Bereich möglich ist", sagte der Professor für Neuroinformatik, der sich hauptsächlich mit Mensch-Maschine-Kommunikation beschäftigt. Professor Dr. Ferdinand Gerlach, Vorsitzender des Sachverständigenrates zur Begutachtung der Entwicklung im Gesundheitswesen, betonte dazu: „Die Diskussion von Delegation und Substitution ist eine Diskussion von gestern. In Zukunft müssen wir vom Prozess her denken - und der heißt: Wie kann ich Patienten versorgen? Und dies erfordert neue Strukturen." 